\title{
Introduction: Time and Technology in Popular Culture, Media and Communication
}

\author{
ADAM GALLIMORE, University of Warwick
}

This special issue of Networking Knowledge addresses several aspects of change in the media by exploring a range of debates about the temporal and the technological across several disciplines and research areas. In doing so, it contributes to the ever-increasing scholarship concerning technological advancement and application, as well as how time is viewed, constructed and experienced.

Due to the persistent drive of technology, issues of technological development and utilisation need constant revision and reassessment. Modernity has progressively been perceived through the demands and implications of time, and technology has had a massive impact on a wide range of time-based media and forms of communication. This issue aims to contribute to larger debates concerning time - such as its representation, experience or perception - by linking it directly to questions of technology.

There are many prominent links between time and technology that relate to issues of change and representation, with technological inventions and innovations providing the potential for new aesthetic, thematic and representational forms of cultural expression. For instance, digital technologies can be seen to have altered the representation of time in film through particular production, editing and exhibition strategies. The way in which time is structured and communicated is dependent on both the technologies used to shape it and the form in which it is expressed. These representational transitions have the potential to transform or alter our perception of time, and therefore how we relate to cultural forms and also to the past itself.

The papers that make up this issue are significant in that, rather than focusing on a single medium or a similar array of texts and timeframes, they open out the field to examine a series of subjects that have often failed to receive sufficient critical or scholarly attention. These include issues of time and technology relating to filmic technologies in documentary cinema, the exploration of memory and nostalgia through video game music, and the significance of representing time within contexts beyond postmodernism. Meanwhile, the growth of social media forums and blogging applications has made it easier for individuals to create their own personal timelines that incorporate and document changing technologies, making it another subject of major contemporary significance. This is addressed through interrogating the limits of digital media to fetishise the past, and through the excavation, recirculation and remediation of cultural waste. This group of papers raises a series of issues, contexts and questions that highlight the importance of these two interconnected concepts. They also represent the dynamic and comprehensive scholarship that is present across several diverse disciplines.

Trent Griffiths examines how the medium of documentary film can become a place for the metaphysical meeting of two filmmakers otherwise separated by time and space. In Grizzly Man (2005), the virtual dialogue that takes place between documentarians Werner Herzog and Timothy Treadwell forms the crux of the film's temporal exploration. Herzog's use of 
Treadwell's footage, Griffiths argues, highlights the complex status of the filmic trace in documentary film, and how these traces can create distinct affective experiences of time. Considering the ethical as well as the structural implications of this practice, Griffiths emphasises the importance of self-reflexivity and re-presentation that is bound up with Herzog's status as author. Technology is key to the quest for self-actualisation for both filmmakers represented in Grizzly Man, foregrounding the complex dimensions of the relationship between documentary time and filmic traces.

Elodie Roy turns her attention to the internet as a site of consumption and waste, an archive of obsolescence and ephemera that forms a unique and expansive collective memory. Acknowledging the fluctuating state of both the archives and the media themselves, Roy suggests that the forms of redemption that are enabled by the internet are strictly inseparable from the production of further layers of cultural waste. The sociality and interactivity of online archives have become familiar to us as web users, yet it is also important to consider the roles they have to play, both in their generation and in their consumption.

Taking the entire career of director Tony Scott into account, Federico Giordano considers how the filmmaker actively adjusted the content and formal structure of his films in terms of depicting contemporaneity. In the central case study, Déjà $V u$ (2006), Giordano finds an exemplary metaphor for the political problems of the epoch, a film that approaches the hypotheses of outdating postmodernism. The importance of technology in the film for surveillance and preventing terrorism is married with the presentation of a non-linear timeline, and this encourages the viewer to make their own inferences and hypotheses. Framed by metamodernism, Giordano sees Déjà $V u$ as neither a traditional film in which forking-path narratives lead to a limited series of alternatives, nor a postmodern film in which all the branches have the same importance; instead, by postulating that viewers should imagine the possible futures of the narrative as 'nearly true', the film can be read as pseudomodernist, a dimension that makes the individual's action the necessary condition of the cultural product.

Andra Ivănescu uses the BioShock series of video games to explore the effect of popular music as a form of narrative engagement. Appropriated music becomes an almost integral part of their games' stories and player experiences, and in BioShock Infinite, pop music of the past, together with an array of anachronistic covers of more modern pop songs, plays an important role at crucial moments in the narrative. Music emerges as a dislocating, disruptive element of the soundscape, literalising the act of time travel as the player moves through the game. Lyrically, the songs become extremely pertinent to the narrative, and Ivănescu illustrates how certain songs (such as Bobby Darin's 'Beyond the Sea') become associated with the game to the extent that players become familiarised with both the world and its music. Appropriated music can often come across as strikingly anachronistic, but Ivănescu argues that the songs work as important temporal signifiers that belong plausibly to the alternate reality of the game. Music also becomes a guide for the player, both directly and indirectly, as well as shaping the extreme contrasts of the dystopia the games represent. Music is thus able to create moments of emotional resonance, evoking emotion as well as memory.

Finally, Elena Caoduro addresses a nostalgic countertrend within contemporary digital media that increasingly values the physical and tactile nature of the analogue image. Taking the online mobile photo-sharing, video-sharing and social networking service Instagram as a central case study, Caoduro considers the effect of the mainstreaming of nostalgia for the 
analogue, and the ways in which the service (and others like it) are able to capitalise on particular aesthetic trends and fetishise technologically obsolete devices, such as lo-fi cameras and vinyl records. Caoduro argues that these new technologies bring back the illusory physicality of picture-taking through digital skeuomorphism, but also questions how this allows users to feel connected to the past by clear and valued signs of age, mimicking a perceived sense of loss. As she states, photo filter applications 'create comfortable memories', bringing with them a different form of personal value that can be related to ritualistic and totemic concepts. While the artistic value of the products are often questioned, criticised for a loss of professionalism and authenticity, Caoduro acknowledges the central paradox of the practice of transforming digital photos with mobile applications, in that it can be both creatively liberating and constraining. The ephemerality of photo filter apps encourages us both to reflect back on the methodology of picture-taking, and also to look forward to future practices, variations and aesthetics.

Each of these papers marks a significant contribution to the broadening studies of time and technology, encouraging us to contemplate our relationships with these two central facets of popular culture, media and communication, as well as considering the potentialities for new directions. Together, this collection of articles provides a sense of the complex, multifarious and rewarding media environment, and the extent to which this has been shaped by the technological and temporal actualities of recent years. 